\title{
Classical Trajectories and Quantum Field Theory
}

\author{
Giuseppe Vitiello \\ Dipartimento di Fisica "E.R.Caianiello", Università di Salerno, 84100 Salerno, Italy \\ INFN, Gruppo Collegato di Salerno and INFM, Sezione di Salerno
}

Received on 11 December, 2004

\begin{abstract}
The density matrix and the Wigner function formalism requires the doubling of the degrees of freedom in quantum mechanics (QM) and quantum field theory (QFT). The doubled degrees of freedom play the role of the thermal bath or environment degrees of freedom and are entangled with the system degrees of freedom. They also account for quantum noise in the fluctuating random forces in the system-environment coupling. The algebraic structure of QFT turns out to be the one of the deformed Hopf algebra. In such a frame, the trajectories in the space of the unitarily inequivalent representations of the canonical commutation relations turn out to be classical trajectories and, under convenient conditions, they may exhibit properties typical of classical chaotic trajectories in nonlinear dynamics. The quantum Brownian motion and the two-slit experiment in QM are discussed in connection with the doubling of the degrees of freedom.
\end{abstract}

\section{INTRODUCTION}

In this paper I consider the problem of the interplay between 'classical and quantum' from the point of view of the intrinsic mathematical structure of Quantum Field Theory (QFT).

The emergence of classicality from Quantum Mechanics $(\mathrm{QM})$ as a result of decoherence has been and is currently analyzed in detail in the literature. One further scenario, since long well known, is the one of the emergence of macroscopic ordered patterns out of a quantum dynamics; this is the case of the generation of classically behaving structures out of QFT (not QM!) with spontaneous breakdown of symmetry. Crystals, ferromagnets, superconductors, superfluids are examples of such classically behaving macroscopic quantum systems. These are quantum systems not in the trivial sense that they, as all other kind of matter, are made of quantum components, but in the sense that their macroscopic behavior, characterized by the classical (c-number) observable called order parameter, cannot be explained without recourse to the underlying quantum field dynamics. On the other hand, the opposite route, from classical to quantum, namely the problem of 'quantization' of a classical theory, is a central problem in many fields of research; paradigmatic examples are the ones of gravitation theories and of non-hamiltonian systems, such as dissipative systems. More recently, an alternative, novel perspective has been proposed [1] (see also [2-4]) for the route from classical to quantum, the one of the 'emergence' of the quantum-like behavior from a classical frame; namely, the possibility has been considered that classical deterministic systems with dissipation (information loss) may exhibit quantum behavior.

My task in this paper is to present and to discuss some results related with aspects of the classical/quantum interplay in connection with the existence in QFT of infinitely many unitarily inequivalent representations (uir) of the canonical commutation (or anti-commutation) relations (ccr).

In QM the well known von Neumann theorem states that for systems with a finite number of degrees of freedom all the representations of the ccr are unitarily equivalent. Such a theorem does not hold in QFT where the systems have infinite number of degrees of freedom. Infinitely many uir of the ccr are thus allowed to exist [5-7] and therefore, in this respect,
QFT is drastically different from QM, and one is not allowed to make confusion among the two. The existence of uir is a characterizing feature of QFT and a full series of physically relevant consequences follows.

In the first part of the paper, by considering some aspects of the quantum Brownian motion and of the two-slit experiment, I discuss the role of the doubling of the degrees of freedom and show how quantum/classical features depend on it (Section II). This part, although physically interesting in itself for the nature of the considered problems, is useful for the subsequent discussion of the deformed Hopf algebra structure [8, 9] of QFT in Section III. This is indeed analyzed in the second part of the paper and leads to recognize that a symplectic structure with classical dynamics is embedded in the space of the uir of ccr in QFT [10]. In this way, a further aspect of the classical/quantum interplay emerges as an intrinsic feature of QFT. The entanglement between the system degrees of freedom and the doubled ones is commented upon in Section IV. In Section V trajectories in the space of the uir are shown to be classical trajectories, which, under convenient conditions, may satisfy the criteria for chaoticity prescribed by nonlinear dynamics. Section VI is devoted to conclusions.

\section{THE DOUBLING OF THE DEGREES OF FREEDOM}

In this Section the main observation is that in QM and in QFT a basic role is played by the doubling of the degrees of freedom of the system under study. It is well known, for example, that in thermal field theory the doubled degrees of freedom describe the heat bath [7, 11-15]; or, near a black hole, such a doubling describes the modes on the two sides of the horizon [16-18].

On a much simpler ground, the familiar operation of adding, e.g., the angular momentum $J^{\alpha}, \alpha=1,2,3$, of two identical particles, is given by $\Delta J^{\alpha}=J^{\alpha} \otimes \mathbf{1}+\mathbf{1} \otimes J^{\alpha} \equiv J_{1}^{\alpha}+$ $J_{2}^{\alpha}$. Such an addition is a homomorphism which indeed duplicates the algebra, $\Delta: \mathcal{A} \rightarrow \mathcal{A} \otimes \mathcal{A}$, i.e. $\Delta O=O \otimes \mathbf{1}+\mathbf{1} \otimes O \equiv$ $O_{1}+O_{2}$, with $O \in \mathcal{A}$. I will come back to this point in the following and the addition operation $\Delta$ will be identified with the coproduct map, thus recognizing the Hopf algebra to be the basic algebraic structure of quantum theory. 
It is much instructive to illustrate the formal and the physical relevance of the doubling of the degrees of freedom by considering some typical problems in QM, such as the two-slit diffraction experiment and the Brownian motion of a quantum particle.

On a formal basis, one can understand the doubling of the degrees of freedom [19-21] by considering the standard expression for the Wigner function [22],

$$
\begin{aligned}
& W(p, x, t)= \\
& \frac{1}{2 \pi \hbar} \int \psi^{*}\left(x-\frac{1}{2} y, t\right) \psi\left(x+\frac{1}{2} y, t\right) e^{\left(-i \frac{p y}{\hbar}\right)} d y .
\end{aligned}
$$

The associated density matrix function is

$$
W(x, y, t)=\left(x_{+}|\rho(t)| x_{-}\right)=\psi^{*}\left(x_{-}, t\right) \psi\left(x_{+}, t\right),
$$

where $x_{ \pm}=x \pm \frac{1}{2} y$. The density matrix and the Wigner function formalism thus requires the introduction of a "doubled" set of coordinates, $\left(x_{ \pm}, p_{ \pm}\right)$(or $\left(x, p_{x}\right)$ and $\left(y, p_{y}\right)$ ).

It is possible to show [20] that the role of the "doubled" $y$ coordinate is absolutely crucial in the quantum regime, since there it accounts for the quantum noise in the fluctuating random force in the system-environment coupling: in the limit of $y \rightarrow 0$ (i.e. for $x_{+}=x_{-}$) quantum effects are lost and the classical limit is obtained. For example, this can be seen by considering the question of how a classical situation with $x_{+}=x_{-}$ arises in the formalism by Schwinger[23] and by Feynman and Vernon [24] for the quantum Brownian motion [21].

In the treatment of quantum Brownian motion one may start by describing a classical object as having a coordinate which depends on time $x(t)$. The density matrix formalism then suggests that a quantum object may be described as splitting the single coordinate $x(t)$ into two coordinates $x_{+}(t)$ (going forward in time) and $x_{-}(t)$ (going backward in time) [23]. The classical limit is obtained when both motions coincide $x(t)=x_{+}(t)=x_{-}(t)$. To see why this is the case, one may employ the Schwinger quantum operator action principle, or recall the mean value of a quantum operator

$$
\begin{gathered}
\bar{A}(t)=(\psi(t)|A| \psi(t))= \\
\iint \psi^{*}\left(x_{-}, t\right)\left(x_{-}|A| x_{+}\right) \psi\left(x_{+}, t\right) d x_{+} d x_{-}= \\
\iint\left(x_{+}|\rho(t)| x_{-}\right)\left(x_{-}|A| x_{+}\right) d x_{+} d x_{-} .
\end{gathered}
$$

Thus one requires the density matrix (2) to follow two copies of the Schrödinger equation, i.e. the forward in time motion and and the backward in time motion, respectively controlled by the two Hamiltonian operators $H_{ \pm}$:

$$
i \hbar \frac{\partial \psi\left(x_{ \pm}, t\right)}{\partial t}=H_{ \pm} \psi\left(x_{ \pm}, t\right)
$$

which gives

$$
i \hbar \frac{\partial\left(x_{+}|\rho(t)| x_{-}\right)}{\partial t}=\mathcal{H}\left(x_{+}|\rho(t)| x_{-}\right)
$$

where

$$
\mathcal{H}=H_{+}-H_{-} .
$$

Working with two copies of the Hamiltonian (i.e. $H_{ \pm}$) operating on the outer product of two Hilbert spaces has been an implicit requirement in quantum mechanics since the very beginning of the theory. For example, from Eqs.(5), (6) one finds immediately that the eigenvalues of the dynamic operator $\mathcal{H}$ are directly the Bohr transition frequencies $\hbar \omega_{n m}=E_{n}-E_{m}$ which was the first clue to the explanation of spectroscopic structure.

The notion that a quantum particle has two coordinates $x_{ \pm}(t)$ moving at the same time is therefore central. It is the difference between the two motions

$$
y=x_{+}-x_{-}
$$

that induces quantum interference. In the following we will show this by explicit calculation of diffraction patterns in the case of the two-slit experiment.

The second step in the description of the Brownian motion of a quantum particle requires to consider the modification of damped evolution operator in Eqs.(5), (6) which becomes, for a Brownian particle of mass $M$ moving in a potential $\mathrm{U}(\mathrm{x})$ with a damping resistance $\mathrm{R},[20,21]$

$$
\begin{gathered}
\mathcal{H}_{\text {Brownian }}=\mathcal{H}-\frac{i k_{B} T R}{\hbar}\left(x_{+}-x_{-}\right)^{2}, \\
\mathcal{H}=\frac{1}{2 M}\left(p_{+}-\frac{R}{2} x_{-}\right)^{2}-\frac{1}{2 M}\left(p_{-}+\frac{R}{2} x_{+}\right)^{2} \\
+U\left(x_{+}\right)-U\left(x_{-}\right) \\
i \hbar \frac{\partial\left(x_{+}|\rho(t)| x_{-}\right)}{\partial t}=\mathcal{H}_{\text {Brownian }}\left(x_{+}|\rho(t)| x_{-}\right)= \\
\mathcal{H}\left(x_{+}|\rho(t)| x_{-}\right)-\left(x_{+}|N[\rho]| x_{-}\right),
\end{gathered}
$$

where $N[\rho] \approx\left(i k_{B} T R / \hbar\right)[x,[x, \rho]]$ describes the effects of the reservoir random thermal noise and the "Hamiltonian" $\mathcal{H}$ the motion in the $\left(x_{+}, x_{-}\right)$plane $[20,21]$.

In Eq. (9) $p_{ \pm}=-i \hbar \frac{\partial}{\partial x_{ \pm}}$. In general the density operator in the above expression describes a mixed statistical state. It can be also shown [21] that the thermal bath contribution to the right hand side of Eq.(8), proportional to fluid temperature T, is equivalent to a white noise fluctuation source coupling the forward and backward motions in Eq.(7) according to

$$
<y(t) y\left(t^{\prime}\right)>_{n o i s e}=\frac{\hbar^{2}}{2 R k_{B} T} \delta\left(t-t^{\prime}\right)
$$

so that thermal fluctuations are always occurring in the difference (7) between forward in time and backward in time coordinates.

The continual occurring of the forward and backward in time motions can also be seen by constructing the forward and backward in time velocities

$$
v_{ \pm}=\frac{\partial \mathcal{H}_{\text {Brownian }}}{\partial p_{ \pm}}= \pm \frac{1}{M}\left(p_{ \pm} \mp \frac{R}{2} x_{\mp}\right) .
$$




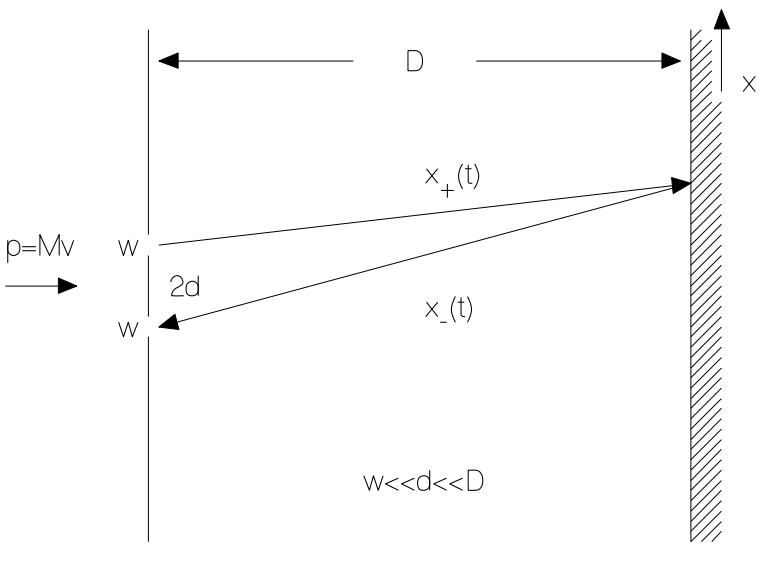

FIG. 1: Two slit experiment.

These velocities do not commute

$$
\left[v_{+}, v_{-}\right]=i \hbar \frac{R}{M^{2}},
$$

and it is thereby impossible to fix the velocities forward and backward in time as being identical. Note that Eq.(13) is similar to the usual commutation relations for the quantum velocities $\mathbf{v}=(\mathbf{p}-(e \mathbf{A} / c)) / M$ of a charged particle moving in a magnetic field $\mathbf{B}$; i.e. $\left[v_{1}, v_{2}\right]=\left(i \hbar e B_{3} / M^{2} c\right)$. Just as the magnetic field $\mathbf{B}$ induces a Aharonov-Bohm phase interference for the charged particle, the Brownian motion friction coefficient $R$ induces a closely analogous phase interference between forward and backward motion which expresses itself as mechanical damping. Eq. (13) has been also discussed in connection with non-commutative geometry in ref.[25]: there it is shown that quantum dissipation induces in the plane of the forward and backward motion a non-commutative geometry.

Let me go now to consider the two-slit diffraction experiment (Fig. 1).

In order to derive the diffraction pattern one needs to know the wave function $\psi_{0}(x)$ of the particle at time zero when it "passes through the slits", or equivalently the density matrix

$$
\left(x_{+}\left|\rho_{0}\right| x_{-}\right)=\psi_{0}^{*}\left(x_{-}\right) \psi_{0}\left(x_{+}\right) .
$$

One wishes to find the probability density for the electron to be found at position $x$ at the detector screen at a latter time $t$,

$$
P(x, t)=(x|\rho(t)| x)=\psi^{*}(x, t) \psi(x, t),
$$

in terms of the solution to the free particle Schrödinger equation which is

$$
\psi(x, t)=\left(\frac{M}{2 \pi \hbar i t}\right)^{1 / 2} \int_{-\infty}^{\infty} e^{\left[\frac{i}{\hbar} A\left(x-x^{\prime}, t\right)\right]} \psi_{0}\left(x^{\prime}\right) d x^{\prime},
$$

where

$$
A\left(x-x^{\prime}, t\right)=\frac{M\left(x-x^{\prime}\right)^{2}}{2 t}
$$

is the Hamilton-Jacobi action for a classical free particle to move from $x^{\prime}$ to $x$ in a time $t$. Eqs.(14)-(17) imply that

$$
\begin{gathered}
P(x, t)= \\
\frac{M}{2 \pi \hbar t} \int_{-\infty}^{\infty} \int_{-\infty}^{\infty} e^{\left[i M \frac{\left(x-x_{+}\right)^{2}-\left(x-x_{-}\right)^{2}}{2 \hbar t}\right]}\left(x_{+}\left|\rho_{0}\right| x_{-}\right) d x_{+} d x_{-} .
\end{gathered}
$$

Then Eq.(18) clearly shows that, were $x_{+}$and $x_{-}$always the same, then $P(x, t)$ not oscillate in $x$, i.e. there would not be the usual quantum diffraction. What is required for quantum interference in Eq.(18) is that the forward in time action $A\left(x-x_{+}, t\right)$ differs from the backward in time action $A\left(x-x_{-}, t\right)$ : The quantum nature of the phenomenon is crucially determined by the non-trivial dependence of the density matrix $\left(x_{+}\left|\rho_{0}\right| x_{-}\right)$when the electron "passes through the slits" on the difference $\left(x_{+}-x_{-}\right)$.

In the usual quantum diffraction experiment one considers $w \ll d \ll D$, where $w$ is the opening of the slits which are separated by a distance $2 d$, and $D$ is the distance between the slits and the screen where the diffraction pattern is observed (see Fig.1). Then the diffraction pattern is well described by $|x| \gg\left|x_{ \pm}\right|$. By defining $K=\frac{M v d}{\hbar D}, \quad \beta=\frac{w}{d}$, with $v=D / t$ the velocity of the incident electron, Eq.(18) leads [21] to the conventional result

$$
P(x, D) \approx \frac{4}{\pi \beta K x^{2}} \cos ^{2}(K x) \sin ^{2}(\beta K x) .
$$

In obtaining (19) the initial wave function

$$
\psi_{0}(x)=\frac{1}{\sqrt{2}}[\phi(x-d)+\phi(x+d)]
$$

with $\phi(x)=\frac{1}{\sqrt{w}}$ if $|x| \leq \frac{w}{2}$ and zero otherwise, has been used. Eqs.(14) and (20) imply that

$$
\begin{gathered}
\left(x_{+}\left|\rho_{0}\right| x_{-}\right)= \\
\frac{1}{2}\left\{\phi\left(x_{+}-d\right) \phi\left(x_{-}-d\right)+\phi\left(x_{+}+d\right) \phi\left(x_{-}+d\right)\right. \\
\left.+\phi\left(x_{+}-d\right) \phi\left(x_{-}+d\right)+\phi\left(x_{+}+d\right) \phi\left(x_{-}-d\right)\right\} .
\end{gathered}
$$

If one accepts the notion of both forward in time and backward in time Hilbert spaces, then the following physical picture of two slit diffraction emerges. The particle can go forward and backward in time through slit 1 (this is described by the first term in the rhs of Eq.(21)). This is a classical process. The particle can go forward in time and backward in time through slit 2, which is also classical since for classical cases $x_{+}(t)=$ $x_{-}(t)$ (the second term in the rhs of Eq.(21)). On the other hand, the particle can go forward in time through slit 1 and backward in time through slit 2 (the third term), or forward in time through slit 2 and backward in time through slit 1 (the fourth term). These are the source for quantum interference since $\left|x_{+}(t)-x_{-}(t)\right|>0$.

In conclusion, by following Schwinger [23], it appears natural to consider doubling the system coordinates, $x(t) \rightarrow$ 
$\left(x_{+}(t), x_{-}(t)\right)$. A system acts in a classical fashion if the two paths can be identified, i.e. $x_{\text {classical }}(t) \equiv x_{+}$classical $(t) \equiv$ $x_{- \text {classical }}(t)$. When the system moves so that the forward in time and backward in time motions are (at the same time) unequal $x_{+}(t) \neq x_{-}(t)$, then the system is behaving in a quantum mechanical fashion and exhibits interference patterns in measured position probability densities. Of course when $x$ is actually measured there is only one classical $x=x_{+}=x_{-}$.

So far I have considered the low temperature limit, which means $T \ll T_{\gamma}$ where $k_{B} T_{\gamma}=\hbar \gamma=\frac{\hbar R}{2 M}$. In the high temperature regime $T \gg T_{\gamma}$, the thermal bath motion suppresses the probability for $x_{+} \neq x_{-}$due to the thermal term $\left(k_{B} T R / \hbar\right)\left(x_{+}-\right.$ $\left.x_{-}\right)^{2}$ in Eq.(8) (cf. also Eq. (11)). By writing the diffusion coefficient $D=\frac{k_{B} T}{R}$ as

$$
D=\frac{T}{T_{\gamma}}\left(\frac{\hbar}{2 M}\right)
$$

the condition for classical Brownian motion for high mass particles is that $D \gg(\hbar / 2 M)$, and the condition for quantum interference with low mass particles is that $D \ll(\hbar / 2 M)$. For large particles in, say, colloidal systems classical Brownian motion would appear to dominate the motion. For a single atom in a fluid at room temperature it is typically $D \sim(\hbar / 2 M)$, equivalently $T \sim T_{\gamma}$ so that quantum mechanics plays an important but perhaps not dominant role in the Brownian motion.

Coordinate doubling has also entered into the canonical quantization of dissipative systems [15, 26, 27] and it appears to be intimately related to the algebraic properties of the theory $[10,12,13,28]$, as I will discuss below.

It is also interesting to note that the "negative" kinematic term in the Hamiltonian (9) also appears in twodimensional gravity models leading to two different strategies in the quantization method [29]: the Schrödinger representation approach, where no negative norm appears, and the string/conformal field theory approach where negative norm states arise as in Gupta-Bleurer electrodynamics.

In the following I will consider the algebraic structure of the space of the physical states emergent from the doubling of the degrees of freedom discussed in the present section.

\section{THE DEFORMED HOPF ALGEBRA IN QFT}

The study of several problems of physical interest where the doubling of the degrees of freedom has proved to play a crucial role in the canonical formalism has suggested, in recent years, that the structure of the state space in QFT is intimately related $[12,13]$ to the one of the deformed Hopf algebra $[8,9]$.

As already observed in the previous Section, the additivity of angular momentum, and of other so-called primitive operators, such as energy and momentum, necessarily implies the use of the coproduct operation, a key ingredient of Hopf algebras, defined by $\Delta O=O \otimes \mathbf{1}+\mathbf{1} \otimes O \equiv O_{1}+O_{2}$, with $O \in \mathcal{A}$, which is a homomorphism which indeed duplicates the algebra, $\Delta: \mathcal{A} \rightarrow \mathcal{A} \otimes \mathcal{A}$. Since additivity of observables is an essential requirement, Lie-Hopf algebra thus appears to be the essential algebraic structure of QM and of QFT. A remarkable result is that the infinitely many uir of the ccr, whose existence characterizes QFT, are classified by use of the deformed Hopf algebra. Quantum deformations of Hopf algebra have thus a deeply non-trivial physical meaning in QFT. One can indeed show $[12,13]$ that the Bogolubov transformations

$$
\begin{aligned}
& A(\theta) \equiv \frac{1}{\sqrt{2}}(\alpha(\theta)+\beta(\theta))=A \cosh \theta-B^{\dagger} \sinh \theta \\
& B(\theta) \equiv \frac{1}{\sqrt{2}}(\alpha(\theta)-\beta(\theta))=B \cosh \theta-A^{\dagger} \sinh \theta
\end{aligned}
$$

are directly obtained by use of the $q$-deformed copodruct operation:

$$
\Delta a_{q}=a_{1} q^{1 / 2}+q^{-1 / 2} a_{2}, \Delta a_{q}^{\dagger}=a_{1}^{\dagger} q^{1 / 2}+q^{-1 / 2} a_{2}^{\dagger} .
$$

In Eqs. (23) and (24) $\alpha(\theta)$ and $\beta(\theta)$ are convenient linear combinations $[12,13]$ of the coproduct operators $(25)$ with the deformation parameter $q=e^{2 \theta}$. Note that $\left[a_{i}, a_{j}\right]=\left[a_{i}, a_{j}^{\dagger}\right]=$ $0, i, j=1,2, i \neq j$ and $\left[A(\theta), A^{\dagger}(\theta)\right]=1, \quad\left[B(\theta), B^{\dagger}(\theta)\right]=1$ and all other commutators equal to zero. $A(\theta)$ and $B(\theta)$ also commute. The momentum suffix $\kappa$ is omitted for simplicity. Note that the $A$ and $B$ (or $a_{1}$ and $a_{2}$ ) operators play here the role of the doubled degrees of freedom $\left(x_{+}\right.$and $x_{-}$in the previous Section).

The generator of (23) and (24) is $\mathcal{G} \equiv-i\left(A^{\dagger} B^{\dagger}-A B\right)$ :

$$
-i \frac{\delta}{\delta \theta} A(\theta)=[\mathcal{G}, A(\theta)], \quad-i \frac{\delta}{\delta \theta} B(\theta)=[\mathcal{G}, B(\theta)],
$$

and h.c.. Thus $p_{\theta} \equiv-i \frac{\delta}{\delta \theta}$ can be regarded $[12,13]$ as the momentum operator "conjugate" to the "degree of freedom" $\theta$. For an assigned fixed value $\bar{\theta}$, it is

$$
e^{\left(i \bar{\theta} p_{\theta}\right)} A(\theta)=e^{(i \bar{\theta} \mathcal{G})} A(\theta) e^{(-i \bar{\theta} \mathcal{G})}=A(\theta+\bar{\theta}),
$$

and similarly for $B(\theta)$.

In the case of time-dependent deformation parameter, the Heisenberg equation for $A(t, \theta(t))$ is

$$
-i \dot{A}(t, \theta(t))=-i \frac{\delta}{\delta t} A(t, \theta(t))-i \frac{\delta \theta}{\delta t} \frac{\delta}{\delta \theta} A(t, \theta(t))=
$$

$$
[H, A(t, \theta(t))]+\frac{\delta \theta}{\delta t}[\mathcal{G}, A(t, \theta(t))]=[H+Q, A(t, \theta(t))],
$$

and $Q \equiv \frac{\delta \theta}{\delta t} \mathcal{G}$ plays the role of the heat-term in dissipative systems. $H$ is the Hamiltonian responsible for the time variation in the explicit time dependence of $A(t, \theta(t)) . H+Q$ can be therefore identified with the free energy [15]: variations in time of the deformation parameter involve dissipation. In thermal theories and in dissipative systems the doubled modes $B$ play the role of the thermal bath or environment.

Let $|0\rangle \equiv|0\rangle \otimes|0\rangle$ denote the vacuum annihilated by $A$ and $B, A|0\rangle=0=B|0\rangle$. By introducing the suffix $\kappa$, at finite volume $V$ one obtains

$$
|0(\theta)\rangle=e^{\left(i \sum_{\kappa} \theta_{\kappa} \mathcal{G}_{\kappa}\right)}|0\rangle=\prod_{k} \frac{1}{\cosh \theta_{k}} e^{\left(\tanh \theta_{k} A_{k}^{\dagger} B_{k}^{\dagger}\right)}|0\rangle .
$$


$\theta$ denotes the set $\left\{\theta_{\kappa}=\frac{1}{2} \ln q_{\kappa}, \forall \kappa\right\}$ and $\langle 0(\theta) \mid 0(\theta)\rangle=1$.

The vacuum $|0(\theta)\rangle$ is an $S U(1,1)$ generalized coherent state [30] (the group structure actually is $\bigotimes_{\kappa} S U(1,1)_{\kappa}$ ): coherence and the vacuum structure in QFT are thus intrinsically related to the deformed Hopf algebra. In the following $\mathcal{H}_{\theta}$ will denote the Hilbert space with vacuum $|0(\theta)\rangle: \mathcal{H}_{\theta} \equiv\{|0(\theta)\rangle\}$. In view of the similarity of some features of the coherent states with those of the fractals, it is an interesting question to ask whether fractal properties enter the QFT structure. A study on this point is in progress.

In the infinite volume limit, the number of degrees of freedom becomes uncountable infinite, hence one obtains $[7,11,15]\left\langle 0(\theta) \mid 0\left(\theta^{\prime}\right)\right\rangle \rightarrow 0 \quad$ as $V \rightarrow \infty, \quad \forall \theta, \theta^{\prime}, \theta \neq \theta^{\prime}$, which means that the Hilbert spaces $\mathcal{H}_{\theta}$ and $\mathcal{H}_{\theta^{\prime}}$ become unitarily inequivalent. In this limit, the "points" of the space $\mathcal{H} \equiv\left\{\mathcal{H}_{\theta}, \forall \theta\right\}$ of the infinitely many uir of the ccr are labelled by the deformation parameter $\theta[12,13,15]$.

Since QFT is characterized by the existence of uir of the ccr [5], and since these uir are related among themselves by the Bogoliubov transformations, which, as seen above are obtained as linear combinations of the deformed coproduct maps, we see that the doubling of the degrees of freedom is a general feature of QFT (independent of the specificity of the system under study) and that the intrinsic algebraic structure of QFT is thus the one of the deformed Hopf algebra. The uir existing in QFT are related and labelled by means of such a algebraic structure.

It should be stressed that the coproduct map is also essential in QM in order to deal with a many modes system (typically, with identical particles). However, in QM all the representations of the ccr are unitarily equivalent and therefore the Bogoliubov transformations induce unitary transformations among the representations, thus preserving their physical content. The deformed Hopf algebra therefore does not have that physical relevance in QM, which it has, on the contrary, in QFT. Here, the representations of the ccr, related through Bogoliubov representations, are unitarily inequivalent and therefore physically inequivalent: they represent different physical phases of the system corresponding to different boundary conditions, such as, for example, the system temperature. Typical examples are the superconducting and the normal phase, the ferromagnetic and the non-magnetic (i.e. zero magnetization) phase, the crystal and the gaseous phase, etc.. The physical meaning of the deformation parameter $q$ in terms of which uir are labelled is thus recognized.

When the above discussion is applied to non-equilibrium thermal field theories it appears that the couple of "thermal" conjugate variables $\theta$ and $p_{\theta} \equiv-i \frac{\partial}{\partial \theta}$, with $\theta=\theta(\beta(t))$ $\left(\beta(t)=\frac{1}{k_{B} T(t)}\right)$, related to the $q$-deformation parameter, describe trajectories in the space $\mathcal{H}$ of the representations, i.e. the space whose "points" are the uir of the ccr $[12,13]$. In [10] it has been shown that there is a symplectic structure associated to the "thermal degrees of freedom" $\theta$ and that the trajectories in the $\mathcal{H}$ space may exhibit some properties typical of chaotic trajectories in classical nonlinear dynamics. Such a picture of a classical nonlinear dynamics in the space $\mathcal{H}$ of the representations is not limited to thermal field theory, but it is a general feature of QFT. We will discuss this in the following.
In the next Section we present further characterizations of the vacuum structure of the uir in QFT.

\section{ENTANGLEMENT AND ENTROPY}

The state $|0(\theta)\rangle$ in Eq. (29) can be written as

$$
\begin{aligned}
& |0(\theta)\rangle=\left(\prod_{k} \frac{1}{\cosh \theta_{k}}\right) \\
& \times\left(|0\rangle \otimes|0\rangle+\sum_{k} \tanh \theta_{k}\left(\left|A_{k}\right\rangle \otimes\left|B_{k}\right\rangle\right)+\ldots\right),
\end{aligned}
$$

which clearly cannot be factorized into the product of two single-mode states. There is thus entanglement between the modes $A$ and $B$ and $|0(\theta)\rangle$ is an entangled state.

The state $|0(\theta)\rangle$ may be also written as:

$$
\begin{aligned}
& |0(\theta)\rangle=\exp \left(-\frac{1}{2} S_{A}\right)|I\rangle=\exp \left(-\frac{1}{2} S_{B}\right)|I\rangle, \\
& S_{A} \equiv-\sum_{\kappa}\left\{A_{\kappa}^{\dagger} A_{\kappa} \ln \sinh ^{2} \theta_{\kappa}-A_{\kappa} A_{\kappa}^{\dagger} \ln \cosh ^{2} \theta_{\kappa}\right\} .
\end{aligned}
$$

In these equations $|I\rangle \equiv \exp \left(\sum_{\kappa} A_{\kappa}^{\dagger} B_{\kappa}^{\dagger}\right)|0\rangle$ and $S_{B}$ is given by an expression similar to $S_{A}$, with $B_{\kappa}$ and $B_{\kappa}^{\dagger}$ replacing $A_{\kappa}$ and $A_{\kappa}^{\dagger}$, respectively. I simply write $S$ for either $S_{A}$ or $S_{B}$. I can also write[7, 11, 15]:

$$
\begin{aligned}
|0(\theta)\rangle & =\sum_{n=0}^{+\infty} \sqrt{W_{n}}(|n\rangle \otimes|n\rangle), \\
W_{n} & =\prod_{k} \frac{\sinh ^{2 n_{k}} \theta_{k}}{\cosh ^{2\left(n_{k}+1\right)} \theta_{k}},
\end{aligned}
$$

with $n$ denoting the set $\left\{n_{\mathrm{K}}\right\}$ and with $0<W_{n}<1$ and $\sum_{n=0}^{+\infty} W_{n}=1$. Then

$$
\left\langle 0(\theta)\left|S_{A}\right| 0(\theta)\right\rangle=\sum_{n=0}^{+\infty} W_{n} \ln W_{n},
$$

and thus $S$ can be interpreted as the entropy operator [7, 11, 15] and it provides a measure of the degree of entanglement. I remark that the entanglement is truly realized in the infinite volume limit where

$$
\langle 0(\theta) \mid 0\rangle=e^{-\frac{V}{(2 \pi)^{3}} \int d^{3} \kappa \ln \cosh \theta_{\kappa}} \underset{V \rightarrow \infty}{\longrightarrow} 0,
$$

provided $\int d^{3} \kappa \ln \cosh \theta_{\kappa}$ is not identically zero. The probability of having the component state $|n\rangle \otimes|n\rangle$ in the state $|0(\theta)\rangle$ is $W_{n}$. Since $W_{n}$ is a decreasing monotonic function of $n$, the contribution of the states $|n\rangle \otimes|n\rangle$ would be suppressed for large $n$ at finite volume. In that case, the transformation induced by the unitary operator $G^{-1}(\theta) \equiv \exp \left(-i \sum_{\kappa} \theta_{\kappa} G_{\kappa}\right)$ could disentangle the $A$ and $B$ sectors. However, this is not the case in 
the infinite volume limit, where the summation extends to an infinite number of components and Eq. (36) holds (in such a limit Eq. (29) is only a formal relation since $G^{-1}(\theta)$ does not exist as a unitary operator)[18].

It is interesting to note that, although the mode $B$ is related with quantum noise effects, nevertheless the $A-B$ entanglement is not affected by such noise effects. The robustness of the entanglement is rooted in the fact that, once the infinite volume limit is reached, there is no unitary generator able to disentangle the $A-B$ coupling.

\section{TRAJECTORIES IN THE $\mathcal{H}$ SPACE}

In this Section I want to discuss the chaotic behavior, under certain conditions, of the trajectories in the $\mathcal{H}$ space.

Let me start by recalling some of the features of the $S U(1,1)$ group structure (see, e.g., [30]).

$S U(1,1)$ realized on $C \times C$ consists of all unimodular $2 \times$ 2 matrices leaving invariant the Hermitian form $\left|z_{1}\right|^{2}-\left|z_{2}\right|^{2}$, $z_{i} \in C, i=1,2$. The complex $z$ plane is foliated under the group action into three orbits: $X_{+}=\{z:|z|<1\}, X_{-}=\{z$ : $|z|>1\}$ and $X_{0}=\{z:|z|=1\}$.

The unit circle $X_{+}=\{\zeta:|\zeta|<1\}, \zeta \equiv e^{i \phi} \tanh \theta$, is isomorphic to the upper sheet of the hyperboloid which is the set $\mathbf{H}$ of pseudo-Euclidean bounded (unit norm) vectors $\mathbf{n}: \mathbf{n} \cdot \mathbf{n}=1$. $\mathbf{H}$ is a Kählerian manifold with metrics

$$
d s^{2}=4 \frac{\partial^{2} F}{\partial \zeta \partial \bar{\zeta}} d \zeta \cdot d \bar{\zeta},
$$

and

$$
F \equiv-\ln \left(1-|\zeta|^{2}\right)
$$

is the Kählerian potential. The metrics is invariant under the group action [30].

The Kählerian manifold $\mathbf{H}$ is known to have a symplectic structure. It may be thus considered as the phase space for the classical dynamics generated by the group action [30].

The $S U(1,1)$ generalized coherent states are recognized to be "points" in $\mathbf{H}$ and transitions among these points induced by the group action are therefore classical trajectories [30] in $\mathbf{H}$ (a similar situation occurs [30] in the $S U(2)$ (fermion) case).

Summarizing, the space of the unitarily inequivalent representations of the ccr, which, as seen in Section III, is the space of the $S U(1,1)$ generalized coherent states, is a Kählerian manifold, $\mathcal{H} \equiv\left\{\mathcal{H}_{\theta}, \forall \boldsymbol{\theta}\right\} \approx \mathbf{H}$; it has a symplectic structure and a classical dynamics is established on it by the $S U(1,1)$ action (generated by $\mathcal{G}$ or, equivalently, by $p_{\theta}: \mathcal{H}_{\theta} \rightarrow \mathcal{H}_{\theta^{\prime}}$ ). Trajectories in $\mathcal{H}$ describe transitions through the representations $\mathcal{H}_{\theta}=\{|0(\theta)\rangle\}$ as the $\theta$-parameter changes, i.e. through the physical phases of the system, the system order parameter being dependent on $\theta$. One may then assume time-dependent $\theta$ : $\theta=\theta(t)$. For example, this is the case of dissipative systems and of non-equilibrium thermal field theories where $\theta_{\kappa}=\theta_{\kappa}(\beta(t))$, with $\beta(t)=\frac{1}{k_{B} T(t)}$.
It is interesting to observe that, considering the transitions $\mathcal{H}_{\theta} \rightarrow \mathcal{H}_{\theta^{\prime}}$, i.e. $|0(\theta)\rangle \rightarrow\left|0\left(\theta^{\prime}\right)\right\rangle$, we have

$$
\left\langle 0(\theta) \mid 0\left(\theta^{\prime}\right)\right\rangle=e^{-\frac{V}{2(2 \pi)^{3}} \int d^{3} \kappa F_{\kappa}\left(\theta, \theta^{\prime}\right)}
$$

where $F_{\kappa}\left(\theta, \theta^{\prime}\right)$ is given by Eq. (38) with $\left|\zeta_{\kappa}\right|^{2}=\tanh ^{2}\left(\theta_{\kappa}-\right.$ $\left.\theta_{\kappa}^{\prime}\right)$, which shows the role played by the Kählerian potential in the motion over $\mathcal{H}$.

The result that the group action induces classical trajectories in $\mathcal{H}$ has been also obtained elsewhere [31, 32] on the ground of more phenomenological considerations.

With reference to the discussion presented in Section II, we may say that on the (classical) trajectories in $\mathcal{H}$ it is $x_{+}=x_{-}=x_{\text {classical }}$, i.e. on these trajectories the quantum noise accounted for by $y$ is fully shielded by the thermal bath (cf. Eq. (11)). In [20] it has been indeed shown that the $y$ freedom contributes to the imaginary part of the action which becomes negligible in the classical regime, but is relevant for the quantum dynamics, namely in each of the "points" in $\mathcal{H}$ (the spaces $\mathcal{H}_{\theta}$ ) through which the trajectory goes as $\theta$ changes. Upon "freezing" the action of $G(\theta)$ (i.e. upon "freezing" the "motion" through the uir) the quantum features of $\mathcal{H}_{\theta}$, at given $\theta$, become manifest. We thus recover 't Hooft picture and the results of ref. [2].

Let me use the notation $|0(t)\rangle_{\theta} \equiv|0(\theta(t))\rangle$. For any $\theta(t)=$ $\left\{\theta_{\kappa}(t), \forall \kappa\right\}$ it is

$$
{ }_{\theta}\langle 0(t) \mid 0(t)\rangle_{\theta}=1, \forall t
$$

I will now restrict the discussion to the case in which, for any $\kappa, \theta_{\kappa}(t)$ is a growing function of time and

$$
\theta(t) \neq \theta\left(t^{\prime}\right), \quad \forall t \neq t^{\prime}, \text { and } \theta(t) \neq \theta^{\prime}\left(t^{\prime}\right), \forall t, t^{\prime} .
$$

Under such conditions the trajectories in $\mathcal{H}$ satisfy the requirements for chaotic behavior in classical nonlinear dynamics. These requirements are the following [33]:

i) the trajectories are bounded and each trajectory does not intersect itself.

ii) trajectories specified by different initial conditions do not intersect.

iii) trajectories of different initial conditions are diverging trajectories

Let $t_{0}=0$ be the initial time. The "initial condition" of the trajectory is then specified by the $\theta(0)$-set, $\theta(0)=$ $\left\{\theta_{\kappa}(0), \forall \kappa\right\}$. One obtains

$$
{ }_{\theta}\left\langle 0(t) \mid 0\left(t^{\prime}\right)\right\rangle_{\theta} \underset{V \rightarrow \infty}{\longrightarrow} 0, \forall t, t^{\prime}, \text { with } t \neq t^{\prime},
$$

provided $\int d^{3} \kappa \ln \cosh \left(\theta_{\kappa}(t)-\theta_{\kappa}\left(t^{\prime}\right)\right)$ is finite and positive for any $t \neq t^{\prime}$.

Eq. (42) expresses the unitary inequivalence of the states $|0(t)\rangle_{\theta}$ (and of the associated Hilbert spaces $\left\{|0(t)\rangle_{\theta}\right\}$ ) at different time values $t \neq t^{\prime}$ in the infinite volume limit. The non-unitarity of time evolution implied for example by the damping is consistently recovered in the unitary inequivalence among representations $\left\{|0(t)\rangle_{\theta}\right\}$ 's at different $t$ 's in the infinite volume limit.

The trajectories are bounded in the sense of Eq. (40), which shows that the "length" (the norm) of the "position vectors" 
(the state vectors at time $t$ ) in $\mathcal{H}$ is finite (and equal to one) for each $t$. Eq. (40) rests on the invariance of the Hermitian form $\left|z_{1}\right|^{2}-\left|z_{2}\right|^{2}, z_{i} \in C, i=1,2$ and I also recall that the manifold of points representing the coherent states $|0(t)\rangle_{\theta}$ for any $t$ is isomorphic to the product of circles of radius $r_{\kappa}^{2}=\tanh ^{2}\left(\theta_{\kappa}(t)\right)$ for any $\kappa$.

Eq. (42) expresses the fact that the trajectory does not crosses itself as time evolves (it is not a periodic trajectory): the "points" $\left\{|0(t)\rangle_{\theta}\right\}$ and $\left\{\left|0\left(t^{\prime}\right)\right\rangle_{\theta}\right\}$ through which the trajectory goes, for any $t$ and $t^{\prime}$, with $t \neq t^{\prime}$, after the initial time $t_{0}=0$, never coincide. The requirement $i$ ) is thus satisfied.

In the infinite volume limit, we also have

$$
{ }_{\theta}\left\langle 0(t) \mid 0\left(t^{\prime}\right)\right\rangle_{\theta^{\prime}} \underset{V \rightarrow \infty}{\longrightarrow} 0 \quad \forall t, t^{\prime} \quad, \forall \theta \neq \theta^{\prime}
$$

Under the assumption (41), Eq. (43) is true also for $t=t^{\prime}$. The meaning of Eqs. (43) is that trajectories specified by different initial conditions $\theta(0) \neq \theta^{\prime}(0)$ never cross each other. The requirement ii) is thus satisfied.

In order to study how the "distance" between trajectories in the space $\mathcal{H}$ behaves as time evolves, consider two trajectories of slightly different initial conditions, say $\theta^{\prime}(0)=\theta(0)+\delta \theta$, with small $\delta \theta$. A difference between the states $|0(t)\rangle_{\theta}$ and $|0(t)\rangle_{\theta^{\prime}}$ is the one between the respective expectation values of the number operator $A_{\kappa}^{\dagger} A_{\kappa}$. For any $\kappa$ at any given $t$, it is

$$
\begin{gathered}
\Delta \mathcal{N}_{A_{\kappa}}(t) \equiv \mathcal{N}_{A_{\kappa}}^{\prime}\left(\theta^{\prime}(t)\right)-\mathcal{N}_{A_{\kappa}}(\theta(t)) \\
={ }_{\theta^{\prime}}\left\langle 0(t)\left|A_{\kappa}^{\dagger} A_{\kappa}\right| 0(t)\right\rangle_{\theta^{\prime}}-{ }_{\theta}\left\langle 0(t)\left|A_{\kappa}^{\dagger} A_{\kappa}\right| 0(t)\right\rangle_{\theta} \\
=\sinh ^{2}\left(\theta^{\prime}{ }_{\kappa}(t)\right)-\sinh ^{2}\left(\theta_{\kappa}(t)\right)=\sinh \left(2 \theta_{\kappa}(t)\right) \delta \theta_{\kappa}(t),
\end{gathered}
$$

where $\delta \theta_{\kappa}(t) \equiv \theta^{\prime}{ }_{\kappa}(t)-\theta_{\kappa}(t)$ is assumed to be greater than zero, and the last equality holds for "small" $\delta \theta_{\kappa}(t)$ for any $\kappa$ at any given $t$. By assuming that $\frac{\partial \delta \theta_{\kappa}}{\partial t}$ has negligible variations in time, the time-derivative gives

$$
\frac{\partial}{\partial t} \Delta \mathcal{N}_{A_{\kappa}}(t)=2 \frac{\partial \theta_{\kappa}(t)}{\partial t} \cosh \left(2 \theta_{\kappa}(t)\right) \delta \theta_{\kappa}
$$

This shows that, provided $\theta_{\kappa}(t)$ is a growing function of $t$, small variations in the initial conditions lead to growing in time $\Delta \mathcal{N}_{A_{\kappa}}(t)$, namely to diverging trajectories as time evolves.

In the assumed hypothesis, at enough large $t$ the divergence is dominated by $\exp \left(2 \theta_{\kappa}(t)\right)$. For each $\kappa$, the quantity $2 \theta_{\kappa}(t)$ could be thus thought to play the role similar to the one of the Lyapunov exponent.
Since [15] $\sum_{\mathrm{\kappa}} E_{\mathrm{\kappa}} \dot{\mathcal{N}}_{A_{\kappa}} d t=\frac{1}{\beta} d S_{A}$, where $E_{\mathrm{\kappa}}$ is the energy of the mode $A_{\kappa}, d S_{A}$ is the entropy variation associated to the modes $A$ and $\dot{\mathcal{N}}_{A_{\kappa}}$ denotes the time derivative of $\mathcal{N}_{A_{\mathrm{K}}}$, the divergence of trajectories of different initial conditions may be expressed in terms of differences in the variations of the entropy (cf. Eqs. (44) and (45)):

$$
\Delta \sum_{\kappa} E_{\kappa} \dot{\mathcal{N}}_{A_{\kappa}}(t) d t=\frac{1}{\beta}\left(d S_{A}^{\prime}-d S_{A}\right) .
$$

The discussion above thus shows that also the requirement iii) is satisfied. The conclusion is that trajectories in the $\mathcal{H}$ space exhibit, under the condition (41) and with $\theta(t)$ a growing function of time, properties typical of the chaotic behavior in classical nonlinear dynamics.

\section{CONCLUSIONS}

Doubling the system degrees of freedom plays a crucial role in QM and QFT. The doubled degrees of freedom describe the thermal bath or environment degrees and are entangled with the system degrees of freedom. They also account for quantum noise in the fluctuating random forces in the system-environment coupling. The algebraic structure of QM and QFT is characterized and generated by the doubling of the degrees of freedom and is the one of the Hopf algebra. The $q$-deformed Hopf algebra allows the labelling of the infinitely many unitarily inequivalent representations of the canonical (anti-)commutation relations existing in Quantum Field Theory and, by means of the Bogoliubov transformations constructed by use of the deformed coproduct operation, transitions among the unitarily inequivalent representations are implemented. These transitions describe the processes of (both quantum and thermal) phase transitions in Quantum Field Theory. The transitions are described by trajectories in the space of the unitarily inequivalent representations, which turns out to be a Kählerian manifold. These trajectory are classical trajectories which under convenient conditions may be chaotic trajectories. In this way we recognize that the classical evolution along these trajectories is naturally embedded in the quantum frame of QFT.

\section{ACKNOWLEDGEMENTS}

I acknowledge partial financial support from Miur, INFN, INFM and COSLAB.
[1] G. 't Hooft, arXiv:hep-th/0003005. G. 't Hooft, Int. J. Theor. Phys., 42 (2003) 355. G. 't Hooft, arXiv:hep-th/0105105.

[2] M. Blasone, P. Jizba and G. Vitiello, Phys. Lett., A 287 (2001) 205.

[3] M. Blasone, E. Celeghini, P. Jizba and G. Vitiello, Phys. Lett.,
A 310 (2003) 393.

[4] H.T. Elze and O. Schipper, Phys. Rev., 66 (2002) 044020.

[5] O. Bratteli and D. W. Robinson, Operator Algebras and Quantum Statistical Mechanics (Springer, Berlin, 1979).

[6] G.L. Sewell, Quantum theory of collective phenomena (Clarendon Press, Oxford 1986). 
[7] H. Umezawa, Advanced field theory: micro, macro and thermal concepts (AIP, N.Y. 1993).

U. Umezawa, M. Matsumoto and M. Tachiki, Thermo Field Dynamics and Condensed States (North-Holland, Amsterdam, 1982)

[8] V. G. Drinfeld, in Proc. ICM Berkeley, CA, ed. by A. M. Gleason (AMS, Providence, R.I., 1986), 798p.

M. Jimbo, Int. J. Mod. Phys., A 4 (1989) 3759.

Yu. I. Manin, Quantum groups and Non-Commutative Geometry (CRM, Montreal, 1988).

[9] E. Celeghini, T. D. Palev and M. Tarlini, Mod. Phys. Lett., B5 (1991) 187

P. P. Kulish and N. Y. Reshetikhin, Lett. Math. Phys., 18 (1989) 143.

[10] G. Vitiello, Int. J. Mod. Phys., B 18 (2004) 785.

[11] Y. Takahashi and H. Umezawa, Collective Phenomena 2 (1975) 55; reprint in Int. J. Mod. Phys. B10 (1996) 1599.

[12] E. Celeghini, S. De Martino, S. De Siena, A. Iorio, M. Rasetti and G. Vitiello, Phys. Lett., A244 (1998) 455.

[13] S. De Martino, S. De Siena and G. Vitiello, Int. J. Mod. Phys., B B 10 (1996) 1615.

[14] E. Celeghini, S. De Martino, S. De Siena, M. Rasetti and G. Vitiello, Annals Phys., 241 (1995) 50.

[15] E. Celeghini, M. Rasetti and G. Vitiello, Annals Phys., 215 (1992) 156

[16] M. Martellini, P. Sodano and G. Vitiello, Nuovo Cim., A 48 (1978) 341

[17] A. Iorio, G. Lambiase and G. Vitiello, Annals Phys. 294 (2001) 234.

[18] A. Iorio, G. Lambiase and G. Vitiello, Annals Phys. 309 (2004) 151.

[19] M.Blasone, P.Jizba and G. Vitiello, in Decoherence and Entropy in complex systems, Lecture Notes in Physics, ed. by H.T. Elze (Springer 2004) p.151, [arXiv:quant-ph/0301031].

[20] Y. N. Srivastava, G. Vitiello and A. Widom, Annals Phys., 238 (1995) 200.

[21] M. Blasone, Y. N. Srivastava, G. Vitiello and A. Widom, Annals Phys., 267 (1998) 61,

[22] R. P. Feynman, Statistical Mechanics (W. A. Benjamin Publ. Co., Reading, Ma. 1972)

[23] J. Schwinger, J. Math. Phys., 2 (1961) 407.

[24] R. P. Feynman and F. L. Vernon, Annals of Phys., 24 (1963) 118 .

[25] S. Sivasubramanian, Y.N. Srivastava, G. Vitiello, A. Widom, Phys. Lett., A 311 (2003) 97,

[26] H. Feshbach and Y. Tikochinsky, Trans. New York Acad. Sci., (Ser.II) 38 (1977) 44.

[27] M. Blasone, E. Graziano, O. K. Pashaev and G. Vitiello, Annals Phys., 252 (1996) 115.

[28] G. Vitiello, Quantum dissipation and coherence, in Quantumlike models and coherent effects, ed. by R.Fedele and P.K.Shukla (World Scientific Publ. Co. Singapore 1995) p. 136, [arXiv:hep-th/9503135].

[29] R. Jackiw, Two lectures on two-dimensional gravity, grqc/9511048; D. Cangemi, R. Jackiw and B. Zwiebach, Annals of Phys., 245 (1996) 408.

[30] A. Perelomov, Generalized Coherent States and Their Applications (Springer, Berlin, 1986).

[31] R. Manka, J. Kuczynski and G. Vitiello, Nucl. Phys., B 276 (1986) 533.

[32] E. Del Giudice, R. Manka, M. Milani and G. Vitiello, Phys. Lett., A 206 (1988) 661.

[33] R. Hilborn, Chaos and nonlinear Dynamics, (Oxford University Press, Oxford, 1994) 